\title{
Optimizing SQL Queries over Text Databases
}

\author{
Alpa Jain ${ }^{\# 1}$, AnHai Doan ${ }^{* 2}$, Luis Gravano ${ }^{\# 3}$ \\ \# Computer Science Department, Columbia University \\ 1 alpa@cs.columbia.edu \\ ${ }^{3}$ gravanodcs . columbia.edu \\ * Department of Computer Sciences, University of Wisconsin-Madison \\ ${ }^{2}$ anhai@cs.wisc.edu
}

\begin{abstract}
Text documents often embed data that is structured in nature, and we can expose this structured data using information extraction technology. By processing a text database with information extraction systems, we can materialize a variety of structured "relations," over which we can then issue regular SQL queries. A key challenge to process SQL queries in this text-based scenario is efficiency: information extraction is timeconsuming, so query processing strategies should minimize the number of documents that they process. Another key challenge is result quality: in the traditional relational world, all correct execution strategies for a SQL query produce the same (correct) result; in contrast, a SQL query execution over a text database might produce answers that are not fully accurate or complete, for a number of reasons. To address these challenges, we study a family of select-project-join SQL queries over text databases, and characterize query processing strategies on their efficiency andcritically - on their result quality as well. We optimize the execution of SQL queries over text databases in a principled, cost-based manner, incorporating this tradeoff between efficiency and result quality in a user-specific fashion. Our large-scale experimentsover real data sets and multiple information extraction systemsshow that our SQL query processing approach consistently picks appropriate execution strategies for the desired balance between efficiency and result quality.
\end{abstract}

\section{INTRODUCTION}

Real-world applications frequently rely on the information in large collections of text documents such as news articles, reports, and email messages. Text often embeds valuable structured data, such as who recommends selling which stocks, who has been hired by which corporation, or the number of people affected by a disease outbreak. In this paper, we consider the problem of effectively answering SQL queries over a collection of text documents.

Applications that benefit from structured data embedded in text arise in a wide range of domains. In disease control, suppose an E. coli outbreak has just occurred in a remote country; an epidemiologist may then want to find the Webaccessible archive of a reputable newspaper in that country, to compile statistics on E. coli outbreaks. In business intelligence, a law firm may want to know if its competitor $X$ has recently worked with a company $Y$, and if so, in which cases; toward this goal, the firm extracts evidence of such cases from the competitor's Web site, recent news articles, and court filings. In scientific data management, a hydrologist may hypothesize that soil erosion near Stevenson City has seriously affected the downstream water quality of the Columbia River, using reports of soil erosion that mention Stevenson City from a variety of sources.

Unfortunately, despite the pervasiveness of these applications, none of the current solutions for addressing the above information needs is fully satisfactory. One solution is to rely on keyword search to retrieve documents that are relevant to the task at hand, and then manually identify the (structured) data of interest in the documents, a tedious process. An alternative is an "extract-then-query" solution, which first converts the text collection into a structured database by applying information extraction (IE) techniques, and then poses SQL queries over the extracted database. Consider again the E. coli example above. Using a suitably trained IE system, our epidemiologist might turn to the (previously unseen) Web-accessible archive of a reputable newspaper from the target country to extract relevant tuples of a relation DiseaseOutbreaks (DiseaseName, Location, Year), where a tuple $\langle d, \ell, y\rangle$ indicates that there was an outbreak of disease $d$ in location $\ell$ in year $y$. Then, the epidemiologist may pose SQL queries over the relation.

This solution can address expressive information needs, but has two important limitations. First, it often wastes substantial time extracting useless information, because only a relatively small number of articles in a text database might be useful to process a query. This is likely the case for the above E. coli query and the newspaper archive. Furthermore, the actual "slice" of the relations that is needed to answer a query (e.g., the tuples with DiseaseName equal to E. coli in our example) may also be relatively small. Then, to answer a query in a timely manner, materializing the entire relations and processing all database documents is undesirable.

Another important limitation of the above solution is that it might be too slow for urgent information needs. Information extraction is well known to be computationally expensive [1] (e.g., applying IE to even a moderate-size collection can easily take many hours for some IE systems). So the above solution would be too slow for an epidemiologist who must act fast to control an infectious disease, or for a stock analyst who must respond to the market in a timely manner. In such cases, reliable but not exhaustive query results might be appropriate, as long as they are returned fast. On the other hand, when the need is not urgent (e.g., as in the law firm and hydrologist examples described earlier), users may want to receive exhaustive query results, for which they may be 
willing to wait a relatively long time. In general, users often have preferences regarding the quality and run-time efficiency expected from the querying process, which the above rigid solution cannot accommodate.

To address the above limitations, in this paper we propose viewing this as a query optimization problem. Accordingly, we define a space of execution plans-which includes the extractthen-query approach - and a cost model that captures user preferences; we also develop a way to estimate plan costs and, correspondingly, to select the best plan. We show that query processing can be decomposed into a sequence of basic steps: retrieving relevant text documents, extracting relations from the documents, cleaning the extracted data, and assembling the cleaned data into the final query answers. Our problem is then to consider the desired user-specified balance between quality and execution efficiency, and choose a strategy accordingly, in a principled, cost-based manner.

In the rest of the paper, we elaborate on our solution to process SQL queries over text databases, called SQOUT (for "SQL queries over unstructured text databases"). Specifically, our contributions are as follows:

- We establish that it is feasible to execute SQL queries over text on-the-fly, with IE systems (Section III).

- We show how IE systems, document retrieval strategies, and data cleaning operators-components often studied in isolation in the past-can be seamlessly integrated to form a space of execution plans for SQL queries over text databases (Section IIII).

- We develop a cost model that exposes the tradeoff between efficiency and result quality, and enables users to flexibly adjust their preferences (Section IV).

- We define the statistics necessary to estimate both execution efficiency and result quality, and show how to obtain such statistics efficiently and effectively from text databases (Sections IV] and V].

- We evaluate our approach with extensive experiments over a real-world data set and using state-of-the-art IE systems. Our results demonstrate that our approach consistently picks execution strategies appropriate for the desired balance between efficiency and result quality (Section VI).

\section{RELATED WORK}

The problem of information extraction from unstructured text has received significant attention (see [2] for a survey). This research has mostly focused on improving IE accuracy (e.g., [3, 4, 5, 6]). The related problem of "wrapper induction" from template-based Web pages has also been studied extensively (e.g., in [7]). In addition, recent work has considered information extraction from the entire Web (e.g., [8]). Recently, [9] introduced a novel technique to leverage existing structured databases for information extraction.

The extraction of information from text is computationally expensive, because of the complex text processing generally required. Approaches have been proposed for improving IE efficiency, to avoid processing useless documents that are not relevant to the extraction task at hand and, instead, focus on promising documents that are likely to contain information to be extracted. One such approach, QXtract [1], uses machine learning to derive keyword queries that identify documents rich in target information. For example, QXtract might derive keyword query [foodborne AND pathogens] to retrieve promising documents for a DiseaseOutbreaks relation.

Recent approaches have also looked into handling extracted data using probabilistic databases, which model data uncertainty by assigning probabilities to tuples. Specifically, [10] showed how to store the output from an extraction system based on Conditional Random Fields in a probabilistic database, after appropriately deriving a probability for each extracted tuple. Recently, [11] introduced a structured query processing system that extracts data from documents in an offline step and relies on probabilistic databases to process user queries. In contrast, our approach handles the extracted data uncertainty during query processing, by resolving extraction errors and data conflicts using data cleaning techniques (Section III]; however, the probabilistic approach in [11] can also be applied towards the same goal, which we will investigate as part of our future work.

The "on-the-fly" extraction nature of our work is somewhat reminiscent of work on question answering (e.g., [12]), but this research focuses on natural language questions, not on SQL queries, and has not considered the efficiency-quality tradeoffs in depth. Other relevant work has focused on specialized scenarios or settings: examples include extraction over dynamic data [13] and combining multiple extraction programs using declarative programs (e.g., UIMA [14], GATE [15], $\mathrm{X} \log$ [16]). Finally, other important extraction aspects besides optimization, such as extraction architecture [17] and schema discovery [18], have also been addressed in the literature.

Closest to this paper is the analysis in [19, 20], which considers (among others) the problem of optimizing the document retrieval strategy for a single IE system $S$. Our current work is related to [19, 20], but differs from it in several crucial aspects. First, [19, 20] has studied only a single-IEsystem scenario; however, to answer practical SQL queries, we must often employ multiple IE systems and relations, which raises novel processing challenges that we will discuss and address in this paper. Second, [19, 20] assumes that $S$ is perfect in that it produces all—and only-correct tuples; we remove this assumption, and develop a principled method to model extraction errors. Finally, unlike [19, 20], we do not optimize for a pre-specified target recall, but consider the goal of balancing recall, precision, and execution time in a flexible manner.

A preliminary, 3-page version of this paper appears in [21].

\section{Problem Statement and Execution Plans}

Consider a document collection $D$, which has been identified for a specific application (e.g., $D$ might be the Webaccessible archive of a newspaper for our E. coli example of

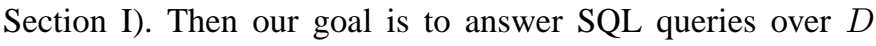
using IE systems. From the many types of IE systems (see 
Section [I], we focus on the common type that takes as input a document and produces as output tuples of the relation for which the system was trained. For instance, consider an IE system trained for a relation Headquarters(Company, Location), where a tuple $\langle c, \ell\rangle$ indicates that $c$ is a company whose headquarters are located in $\ell$. Then, from the text snippet, "Redmond-based Microsoft announced today ..." of a document, this IE system may extract tuple $\langle$ Microsoft, Redmond $\rangle$.

In this paper, we focus on the problem of processing SQL queries using such IE systems:

Problem Statement 1: Consider a text database $D$ with a boolean search interface [22], and $n$ "base" relations $R_{1}, \ldots, R_{n}$ defined over $D$. Each base relation $R_{i}$ can be extracted from $D$ using one or more IE systems. We assume that all base relations $R_{1}, \ldots, R_{n}$ share the same primary key $K$ and no other attributes, so each relation might be regarded as contributing additional attributes of the entities identified by the key attributes in $K$. We define a view $V=\left(R_{1} \bowtie \cdots \bowtie\right.$ $\left.R_{n}\right)_{K}$ as the natural outerjoin of the base relations $R_{1}, \ldots, R_{n}$ over the $K$ attributes. We consider SQL selection-projection queries over $V$ with selection condition conjuncts of the form $A=t$, where $A$ is a textual attribute and $t$ is a constant. Then, given such a SQL selection-projection query $Q$ over $V$, our goal is to identify an execution strategy for $Q$ that meets the desired efficiency and result quality requirements.

Following this problem statement, consider an IE system trained to extract the Headquarters relation above, as well as another system trained for an Executives(Company, CEO) relation, where a tuple $\langle c, e\rangle$ indicates that person $e$ is the CEO of company $c$. We can then define a view CompanyInfo(Company, Location, CEO) as the natural outerjoin of these two "base" relations and express queries such as:

Q1: SELECT Company, CEO FROM CompanyInfo WHERE Location $=$ 'Redmond'

This problem setting is appropriate as a first step in our investigation of the general problem of answering SQL queries over text databases, and is already useful in practice (see Section VI-B. In Section VII we discuss how to generalize our approach further.

Given a SQL query $Q$ as described above, we now discuss the space of execution plans for $Q$. To evaluate $Q$ over database $D$, we need to:

(1) Select an IE system $E_{i}$ for each base relation $R_{i}$.

(2) Select a document retrieval strategy $X_{i}$ for each $E_{i}$.

(3) Use each strategy $X_{i}$ to retrieve from database $D$ a set of documents $P_{i}$, then process $P_{i}$ using IE system $E_{i}$ to obtain a relation instance $r_{i}$.

(4) Clean the relations $r_{1}, \ldots, r_{n}$ by reconciling references and eliminating data inconsistencies.

(5) Execute $Q$ over view $v=\left(r_{1} \bowtie \cdots \bowtie r_{n}\right)_{K}$.

We now elaborate on these steps.

Step (1), Selecting IE Systems: Given a query $Q$, we first determine the base relations that are needed for $Q$, based on the attributes in the SELECT and WHERE clauses. This, in turn, identifies the IE systems that are relevant to $Q$. If more than one IE system is available for a certain base relation, then which system we choose will depend on their efficiency and quality as well as on the user preferences (see Section IV].

Step (2), Selecting Retrieval Strategies: For each IE system $E$, we select a way to retrieve the documents that $E$ will process. (This is analogous to selecting an access path in an RDBMS.) We consider four representative strategies based on the existing literature [19, 20]:

Scan: We sequentially scan the collection and feed each document to $E$. This strategy produces the complete query results for $E$, but makes $E$ process all documents.

Const: We find constants (if any) in query $Q$, then feed $E$ only the documents that contain those constants. For example, the constant "Redmond" in query $Q 1$ above can be used as a keyword query to retrieve only documents with this word for Headquarters, on the ground that documents without it could not contribute useful tuples for that base relation. Const thus avoids processing all documents, and its efficiency is determined by the selectivity of the constants in the query.

PromD: Given E, PromD employs the learning method of QXtract [1] to derive keyword queries (e.g., [based AND shares] for Headquarters) and feed $E$ only "promising" documents that contain these keywords. Thus, like Const, PromD also avoids processing all documents. However, PromD may miss some answer tuples and is IE-system-dependent, in that the keyword queries that it derives-and thus the set of documents retrieved-depend on the IE system $E$.

PromC: This strategy combines Const and PromD by ANDing their queries, to retrieve documents that are promising and, at the same time, satisfy the predicates of the SQL query. For example, for $Q 1$ we combine query [based AND shares] from PromD with query [Redmond] from Const to obtain query [based AND shares AND Redmond]. Similarly to PromD, Prom C avoids processing all documents, but may miss some answer tuples and is IE-system-dependent.

The following example illustrates these strategies:

Example 3.1: Figure 1 shows a possible execution for query Q1. This execution employs two document retrieval strategies, PromC for Headquarters and Scan for Executives. PromC issues queries such as [based AND shares AND Redmond] to the search interface of the database, to retrieve promising documents. After feeding each of these documents to the Headquarters IE system, we obtain tuples such as $\langle$ Microsoft, Redmond $\rangle$. Note that $\langle$ Microsoft Corp., New York $\rangle$ was (erroneously) extracted in this step by the (often-less-than-perfect) extraction system. To extract Executives, Scan retrieves all documents exhaustively, one at a time, and feeds them to the extraction system for this relation, to extract tuples such as $\langle$ Microsoft Corp., Bill Gates $\rangle$.

When query $Q$ has a selection condition associated with a base relation, then all four retrieval strategies are applicable 


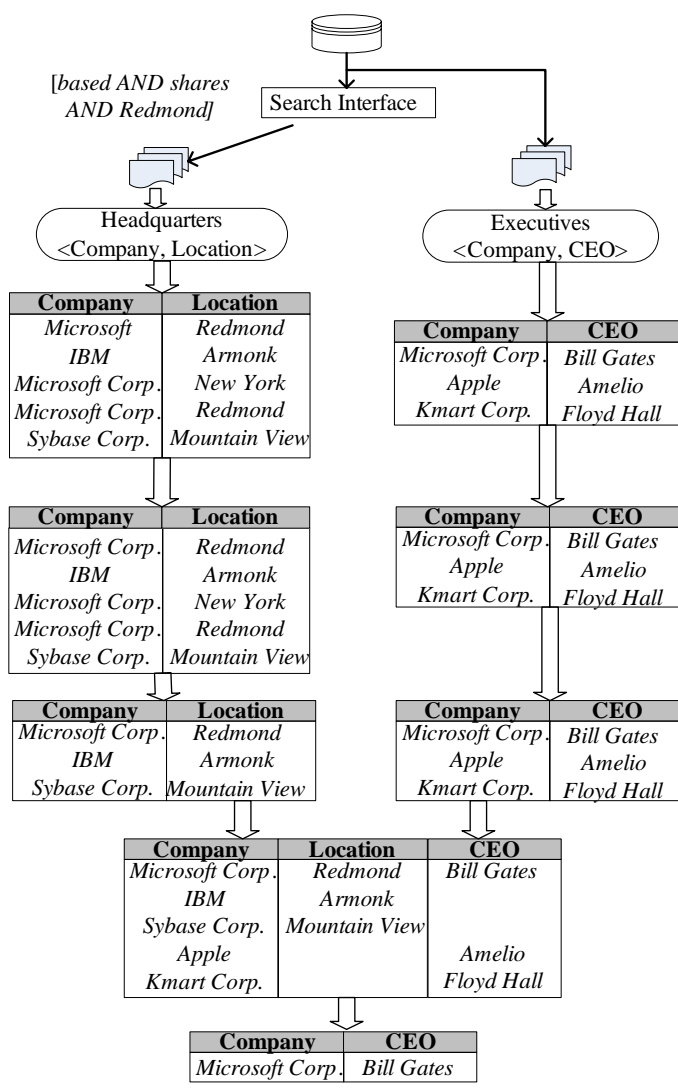

Fig. 1. Stages in the execution of query Q1.

for the relation (e.g., this is the case for Headquarters in query Q1). However, in such case Const is preferable to Scan: Const returns all documents that can contribute to the query result and never processes more documents than Scan. Similarly, Prom C is preferable to PromD. Hence, in this case we will consider only Const and PromC for retrieving documents for the base relation. If, in contrast, a base relation has no associated selection condition, then only Scan and PromD apply.

Step (3), Retrieving and Processing Documents: Upon identifying the documents to process for each IE system $E$, we retrieve these documents from disk, feed them to $E$, and write the extracted tuples to an auxiliary database, for data cleaning and further query processing.

Step (4), Cleaning Extracted Data: The extracted tuples are often noisy: typographical errors commonly occur in text, entity references might be far from uniform, IE systems are error-prone, and text databases might sometimes include contradictions, which would be problematic even in the absence of extraction errors.

To address this problem, we first reconcile entity references. In principle, we can apply any existing reference reconciliation technique (variants of which are also known as record linkage, among other terms [2, 23]). For now, we rely on the domain- independent technique of $[24]^{1}$, which is effective and can be implemented robustly within a RDBMS. We apply this technique to the values of the key attributes $K$ of all our extracted relations collectively. For example, in Figure 11, we conclude that "Microsoft" and "Microsoft Corp." refer to the same company, in both Headquarters and Executives. We then pick an arbitrary canonical representation for the entity from among the associated values. For now, we choose the longest string in each group, so that "Microsoft Corp." represents "Microsoft."

We then resolve semantic inconsistencies due to extraction errors and inherent inconsistencies in the data. Rather than attempting to do this in a sophisticated way, we focus on simple majority arguments. Specifically, we group the extracted tuples in each relation (after reference reconciliation) by their key attributes and choose the most frequent value in each group, individually for each non-key attribute. The rationale is that text databases often exhibit substantial redundancy [3, 4] and errors are likely to be outnumbered by correct facts. Of course, alternate approaches could be used, especially in the presence of domain knowledge, and such approaches could be immediately substituted in our algorithm.

Step (5), Processing Query over Extracted View: As a final step, we execute the SQL query over the cleaned, materialized view generated after the above steps.

To summarize, we explore a space of candidate execution plans by seamlessly combining multiple IE systems along with appropriately chosen document retrieval strategies, and data cleaning techniques. Next, we discuss our cost model, to characterize query execution plans in terms of their efficiency and result quality.

\section{EFFiciency-vs.-Quality Cost Model}

To compare alternate execution strategies for a query $Q$ over a database $D$, we will define the goodness of a query execution as a function of its efficiency and result quality. For this, we define efficiency as follows:

Definition 4.1: [Efficiency] The efficiency of a query execution $S$ over a text database $D, E(S, D)$, is the inverse of the execution time of $S$ over $D$.

The goodness of an execution strategy is also a function of its result quality. So, we need to characterize the "ideal" result for $Q$ over $D$, Ideal $(Q, D)$. This hypothetical ideal result consists of all the correct tuples for $Q$ that could be derived from database $D$ by "perfect" IE systems (i.e., IE systems with perfect precision and recall), using the Scan document retrieval strategy (i.e., by processing all database documents exhaustively), and by fully cleaning the data. Based on Ideal, we define precision and recall as follows:

Definition 4.2: [Precision and Recall] Consider an execution strategy $S$ for a query $Q$ over a text database $D$, and let $R$ be the results that $S$ produces. We define the precision

${ }^{1}$ http://pages.stern.nyu.edu/ 〜panos/datacleaning 
of $S$ over $D$ as $P(S, D)=\frac{\mid R \cap I \text { deal }(Q, D) \mid}{|R|}$ and the recall of $S$ over $D$ as $R(S, D)=\frac{|R \cap \operatorname{Ideal}(Q, D)|}{|\operatorname{Ideal}(Q, D)|}$.

Of course, $|\operatorname{Ideal}(Q, D)|$ is prohibitively expensive to compute for any large database $D$, since this "computation" would necessarily involve substantial human effort (e.g., no "perfect" IE systems exist). So we need to avoid computing Ideal when we characterize the goodness of an execution strategy. A key observation is that, to choose between strategies $S_{1}$ and $S_{2}$, with goodness values $g_{1}$ and $g_{2}$, respectively, we do not need to consider the exact values for $g_{1}$ and $g_{2}$. Instead, it suffices to inspect their ratio $\frac{g_{1}}{g_{2}}$ to decide which strategy is best. With this observation in mind, we define goodness-and quality, a metric based on which goodness is specified-so that the $|\operatorname{Ideal}(Q, D)|$ constant in the denominators of both $g_{1}$ and $g_{2}$ "cancels out" when we consider goodness ratios. Specifically, we combine precision and recall into a single metric by computing their geometric mean, as follows

Definition 4.3: [Quality] Consider an execution strategy $S$ for a query $Q$ over a text database $D$. We define the quality of $S$ over $D$ as $Q(S, D)=(P(S, D) \cdot R(S, D))^{1 / 2}$.

Our definition of quality is similar in spirit to the $F_{1}$ measure [22] used in information retrieval, yet our metric is easier to estimate in the realistic scenario where the Ideal results are not available, as we will see.

To finally define goodness, we note once again that, ultimately, the choice of the right balance between efficiency and quality is user-specific: sometimes users desire high-quality exhaustive query results, even if query execution takes a relatively long time; some other times, users are after some "quick and dirty" answers. We capture this desired efficiency-quality balance with a (user-specified) query processing parameter $w$, ranging from 0 , to privilege efficiency, to 1 , to privilege result quality. In turn, we use parameter $w$ to characterize the overall goodness of a query execution, as follows:

Definition 4.4: [Goodness] The goodness of a query execution $S$ over a text database $D$ for user-specified parameter $w$ is $G_{w}(S, D)=Q(S, D)^{w} \cdot E(S, D)^{(1-w)}$.

The goodness function, as defined above, captures (a weighted version of) the result quality per execution time unit, and allows users to weigh efficiency and result quality appropriately. Several alternate query paradigms can also be used to reflect the user preferences. In particular, we could follow the relational model and define a STOP-AFTER-K-TUPLES paradigm, where the objective of an execution is to derive only $K$ answer tuples as efficiently as possible. Alternatively, a STOP-AFTER-TIME-T paradigm could indicate that the query execution must finish within $T$ time units and produce the highest quality results possible within that time frame. In this paper, we focus on finding query execution strategies with highest goodness, as in Definition 4.4, we will investigate the above alternate query paradigms — and others-in our future

\footnotetext{
${ }^{2}$ Our definition of quality weighs precision and recall equally. This definition can be easily generalized, though, to allow for different (user-specified) weights for precision and recall.
}

\begin{tabular}{ll}
\hline Symbol & Description \\
\hline$|\operatorname{Docs}(E, X, D)|$ & $\begin{array}{l}\text { number of documents retrieved from } D \text { by } \\
\text { retrieval strategy } X \text { for extraction system } E\end{array}$ \\
\hline $\operatorname{RTime}(E, X, D)$ & $\begin{array}{l}\text { average time to retrieve a document from } D \\
\text { using } X \text { for extraction system } E\end{array}$ \\
\hline $\operatorname{ETime}(E, X, D)$ & $\begin{array}{l}\text { average time to run } E \text { on a document retrieved } \\
\text { from } D \text { using } X\end{array}$ \\
\hline$|T(E, X, D)|$ & $\begin{array}{l}\text { average number of tuples that } E \text { extracts from } \\
\text { a document retrieved from } D \text { using } X\end{array}$ \\
\hline$|C(E, X, D)|$ & $\begin{array}{l}\text { average number of correct tuples that } E \text { extracts } \\
\text { from a document retrieved from } D \text { using } X\end{array}$ \\
\hline$|\operatorname{Join}(\mathcal{E}, D)|$ & $\begin{array}{l}\text { cardinality of the join of relations } R_{1}, \ldots, R_{n}, \\
\text { extracted as specified in } \mathcal{E}=\left\{\left(E_{1}, X_{1}\right), \ldots,\right. \\
\left.\left(E_{n}, X_{n}\right)\right\}, \text { where } E_{i} \text { is an extraction system for } R_{i}, \\
\text { with document retrieval strategy } X_{i}(i=1, \ldots, n)\end{array}$ \\
\hline
\end{tabular}

TABLE I

DATABASE-SPECIFIC STATISTICS.

research work.

To estimate the properties of each candidate execution strategy, we use the statistics in Table I for an IE system $E$ with an associated document retrieval strategy $X$ (i.e., Scan, PromD, Const, or PromC) over a database D. (In Section V. we describe how we gather the Table I 1 statistics.) Note that the behavior of $X$ may be dependent on the choice of IE system $E$ (e.g., as is the case for PromD; see Section IIII), so statistics such as $|\operatorname{Docs}(E, X, D)|$ depend not only on $X$ and $D$, but also on $E$ (Table I).

Single-Relation Queries: Consider a query execution strategy $S$ over a database $D$, and assume that $S$ involves only one extraction system, $E$, with an associated retrieval strategy $X$. Our efficiency analysis ignores the post-extraction processing of Steps (4) and (5): the time required for these steps is directly proportional to the number of tuples extracted, and this time is (indirectly) accounted for by the analysis for Step (3), which considers the number of documents processed. Then, we estimate the execution time for $S$ over $D$ as:

$|\operatorname{Docs}(E, X, D)| \cdot(R T i m e(E, X, D)+\operatorname{ETime}(E, X, D))$

The estimated efficiency of $S, E_{\text {est }}(S, D)$, then follows directly from this expression. We estimate precision as:

$$
P_{e s t}(S, D)=\frac{|C(E, X, D)|}{|T(E, X, D)|}
$$

To estimate recall, we face the challenge that $|\operatorname{Ideal}(Q, D)|$ is unknown in the denominator of the definition of recall (Definition 4.2 and computing it would require a prohibitively large human effor ${ }^{3}$ We observe that the $|\operatorname{Ideal}(Q, D)|$ factor affects the absolute goodness value (Definition 4.4) of the executions, but not their relative standing, as discussed earlier in this section. So we can safely replace recall with $R_{e s t}(S, D)=|C(E, X, D)| \cdot|\operatorname{Docs}(E, X, D)|:$ this value is the

\footnotetext{
${ }^{3}$ This problem is analogous to computing the exact recall for information retrieval techniques, an impossible proposition over large data sets.
} 
estimated number of correct tuples extracted by $E$ and $X$ from $D$. Equivalently, using Equation (2),

$R_{e s t}(S, D)=P_{\text {est }}(S, D) \cdot|T(E, X, D)| \cdot|\operatorname{Docs}(E, X, D)|$

Multiple-Relation Queries: We now focus on queries that join multiple base relations. Specifically, consider a query execution strategy $S$ over a database $D$, involving extraction systems $E_{1}, \ldots, E_{n}$, with associated document retrieval strategies $X_{1}, \ldots, X_{n}$, respectively. We estimate the execution time for $S$ as the sum of the time to generate each base relation, computed using Equation (1):

$\sum_{i=1}^{n}\left|\operatorname{Docs}\left(E_{i}, X_{i}, D\right)\right| \cdot\left(\operatorname{RTime}\left(E_{i}, X_{i}, D\right)+\operatorname{ETime}\left(E_{i}, X_{i}, D\right)\right)$

Estimating precision for strategies that involve multiple extraction systems - and hence, extract multiple base relationsis challenging. In absence of additional information, we can assume independence for the extraction errors across the relations and define precision based on the values for the individual relations, as $P_{\text {est }}(S, D)=\prod_{i=1}^{n} \frac{\left|C\left(E_{i}, X_{i}, D\right)\right|}{\left|T\left(E_{i}, X_{i}, D\right)\right|}$. However, we observed experimentally that this assumption can result in underestimating the true precision, so we "boost" the singlerelation precision before proceeding with the independence assumption and define:

$$
P_{\text {est }}(S, D)=\prod_{i=1}^{n} \frac{2 \cdot P_{i}}{1+P_{i}}
$$

where $P_{i}=\frac{\left|C\left(E_{i}, X_{i}, D\right)\right|}{\left|T\left(E_{i}, X_{i}, D\right)\right|}$ and $n \geq 2$.

Regarding recall, we proceed as in the single-relation case (Equation (3) ) and substitute the total number of correct tuples generated by $S$. Thus,

$R_{e s t}(S, D)=P_{e s t}(S, D) \cdot\left|\operatorname{Join}\left(\left\{\left(E_{1}, X_{1}\right), \ldots,\left(E_{n}, X_{n}\right)\right\}, D\right)\right|$

where $\left|\operatorname{Join}\left(\left\{\left(E_{1}, X_{1}\right), \ldots,\left(E_{n}, X_{n}\right)\right\}, D\right)\right|$ is the number of tuples in the (inner) join of the relations extracted from $D$ by extraction systems $E_{1}, \ldots, E_{n}$, with respective document retrieval strategies $X_{1}, \ldots, X_{n}$. We estimate the join cardinality by appropriately scaling, in turn, the cardinality of the corresponding join computed over a document sample, as we describe in Section $\mathrm{V}-\mathrm{B}$

By substituting $E_{e s t}, P_{e s t}$, and $R_{e s t}$ for $E, P$, and $R$ in Definitions 4.3 and 4.4 . we can obtain a "proxy" goodness value whose computation does not involve knowledge of $|\operatorname{Ideal}(Q, D)|$, as discussed. As we argued above, the strategy with highest "proxy" goodness also has highest actual goodness, so we can optimize the execution of a query effectively and without human intervention.

\section{Deriving Database Statistics}

We now discuss how to estimate the statistics in Table [1. which requires that we resort to document sampling in a preprocessing step for SQOUT. We determine the sample size via the sequential sampling method in [25, 26] to achieve an appropriate confidence level on our statistics estimates. In our experiments, we target a 95\% confidence level, which requires independent sample sizes of up to $5 \%$ of the collection size. In general, we can compute the statistics for Scan and PromD offline, while Const and PromC, which depend on queryspecific constants, require some processing at query time.

\section{A. Document Retrieval Statistics}

Consider an IE system $E$ and a database $D$. The document retrieval statistics on which we rely are:

Number of documents retrieved: Scan always retrieves all database documents, so $|\operatorname{Docs}(E, \operatorname{Scan}, D)|=|D|$. PromD retrieves only the documents that match its associated queries, so we derive $|\operatorname{Docs}(E, \operatorname{Prom} D, D)|$ 一offline-by issuing the PromD queries associated with extraction system $E$ to the database $D$ and counting the unique documents among the query matches. Unlike Scan and PromD, Const and PromC retrieve documents in a query-specific manner, since these retrieval strategies rely on the constants in the SQL query. We can efficiently derive $\mid \operatorname{Docs}(E$, Const, $D) \mid$ at query-processing time by issuing the SQL constants as queries to the database (e.g., [Redmond] for our example SQL query Q1) and extracting the number of matches that the queries generate, without retrieving any documents.

To compute $\mid \operatorname{Docs}(E$, PromC, $D) \mid$, we know (1) the number of matches-computed offline-for the PromD queries and (2) the number of matching documents-computed at queryprocessing time-for the Const queries. The number of documents that match the conjunction of each PromD query and each Const query is unknown, however, and issuing these queries to the database at query-processing time would be too expensive. Instead, we analyze-in an offline training step-whether database constants associated with each relation attribute tend to strongly co-occur with the PromD query keywords in the database documents ${ }^{4}$ If this is the case, then we assume that each PromC query, formed by the conjunction of a PromD query and a Const query, returns the minimum of the number of matches of each individual query. Otherwise, we estimate $|\operatorname{Docs}(E, \operatorname{Prom} C, D)|$ - by assuming independenceas $\mid \operatorname{Docs}(E$, Const, $D)|\cdot| \operatorname{Docs}(E, \operatorname{PromD}, D)|/| D \mid$.

Retrieval time: We estimate RTime $(E$, Scan, D)—offlineby computing the time required to retrieve each document in a random sample from $D$. We use this same estimate for RTime(E, Const, D), since the Const queries tend to be short and hence relatively inexpensive to process. For PromD, the retrieval time is estimated-also offline-over a sample of the documents that match the PromD queries associated with $E$. We use this same estimate for $\operatorname{RTime}(E$, Prom C, D), since the PromC queries tend to be only slightly longer than the PromD queries, and hence their retrieval-time behavior is similar.

\section{B. Extraction Statistics}

Consider an extraction system $E$ with an associated document retrieval strategy $X$ over a database $D$. To estimate

\footnotetext{
${ }^{4}$ This analysis relies on a paired $t$-test [27] and other statistical methods, which we do not discuss further because of space limitations.
} 
the extraction statistics in Table I], we consider the (tuple-rich) documents that match the PromD queries for $E$ separately from the rest of the documents: these two subsets of $D$ are likely to exhibit substantially different values for the extraction statistics. Specifically, we perform stratified sampling [27] over $D$, with one stratum, $P_{D}$, corresponding to the PromD matches in $D$ and the other stratum corresponding to the remainder of the documents in $D$. Figure 2 shows these strata for $D$. For Scan, the extraction statistics below are computed over both strata, namely $P_{D}$ and $D-P_{D}$. For PromD and PromC, we compute the statistics over $P_{D}$ only. Finally, for Const we use both $P_{D}$ and $D-P_{D}$ : conceptually, Const retrieves all of the PromC documents, which are included in $P_{D}$, plus some additional documents, which are in $D-P_{D}$ (see Figure 2). We compute the extraction statistics for Const based on $\mid \operatorname{Docs}(E$, $\operatorname{PromC}, D)|,| \operatorname{Docs}(E$, Const, D)|, and the stratified sample statistics.

For instance, when deriving $|T(E, S c a n, D)|$ as described above, we retrieve documents until we reach a confidence of $95 \%$ in the estimated value. Then, if $E$ extracted, on average, say $t_{D}$ tuples from the $D-P_{D}$ stratum and $t_{P_{D}}$ tuples from the $P_{D}$ stratum, we compute $|T(E, S c a n, D)|$ as a weighted average of $t_{D}$ and $t_{P_{D}}$, with weights $\left|D-P_{D}\right|$ and $\left|P_{D}\right|$, respectively. The other statistics are handled analogously.

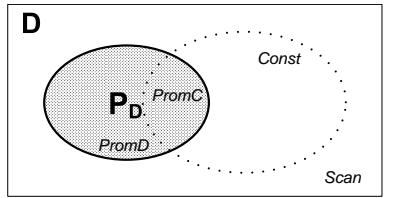

Fig. 2. Database strata for sampling.

Extraction time and number of extracted tuples: We estimate $\operatorname{ETime}(E, X, D)$ and $|T(E, X, D)|$ by running $E$ over the document sample(s) for $X$ (see above).

Join cardinality: $|\operatorname{Join}(\mathcal{E}, D)|$, for $\mathcal{E}=\left\{\left(E_{1}, X_{1}\right), \ldots\right.$, $\left.\left(E_{n}, X_{n}\right)\right\}$, is the cardinality of the join of the extracted relations $R_{1}, \ldots, R_{n}$, where $R_{i}$ is extracted using extraction system $E_{i}$ and document retrieval strategy $X_{i}(i=1, \ldots, n)$. To estimate this cardinality, we handle the Scan and PromD cases offline: we first focus on the appropriate document sample for each $E_{i}, X_{i}$ pair, as discussed above, then extract the relation tuples, $\operatorname{Sample}\left(E_{i}, X_{i}, D\right)$, from each document sample, and finally join the extracted relations to determine the sample join cardinality $j_{s}$. Then, we produce the $|\operatorname{Join}(\mathcal{E}, D)|$ estimate as [28]:

$$
|\operatorname{Join}(\mathcal{E}, D)|=j_{s} \cdot \prod_{i=1}^{n} \frac{\left|T\left(E_{i}, X_{i}, D\right)\right| \cdot\left|\operatorname{Docs}\left(E_{i}, X_{i}, D\right)\right|}{\left|\operatorname{Sample}\left(E_{i}, X_{i}, D\right)\right|}
$$

For Const and PromC, we estimate the join cardinality online, from the corresponding join cardinality values $j_{s}$ for Scan and PromD, respectively, as discussed above.

Verifying tuple correctness: To compute $|C(E, X, D)|$, we need to decide whether extracted tuples are correct, for which we could manually inspect the text database to make a decision. This manual inspection is, of course, tedious and prohibitively time-consuming, so we resort instead to a more automatic "verification" approach. Specifically, we first manually define a small number of "safe" natural-language patterns for each relation, such as "〈LOCATION〉-based 〈ORGANIZATION〉" for Headquarters. To decide whether an extracted tuple is correct or not, we instantiate the safe patterns for the relation with the attribute values for the tuple, and search for instances of the instantiated patterns using the database's search interface. Intuitively, we automate the process of deriving statistics by simulating a manual evaluation of tuples. For example, tuple 〈Microsoft Corp., Redmond $\rangle$ results in an instantiated pattern "Redmond-based Microsoft Corp." We consider the presence of an instantiated pattern in a database as strong evidence of tuple correctness, from which we derive a conservative approximation of the number of correct tuples extracted by an extraction system.

\section{EXPERIMENTAL EVALUATION}

We now describe the settings for our experiments and report the experimental results.

\section{A. Experimental Settings}

Data and relations: We use a subset of the North American News Text Corpus 5 with 1995-6 articles from The New York Times, split into a training (135,438 documents from 1996) and a test database (137,893 documents from 1995). We define two "base" relations, Headquarters(Company, Location) and Executives(Company, CEO), and a view CompanyInfo(Company, Location, CEO) over the base relations.

Queries: We use 33 SQL queries over CompanyInfo, defined manually to cover an interesting mix of selections (e.g., SELECT * FROM CompanyInfo WHERE Location = 'California') and projections (e.g., SELECT Company, CEO FROM CompanyInfo), including selections with many and with few or no database matches.

IE systems: We trained variations of our home-grown implementations of DIPRE [4] and Snowball [3] for Headquarters and Executives. We modified the original formulation of DIPRE to fit our newspaper database (e.g., we do not exploit URLs or HTML tags, but incorporate instead named-entity tags, such as $\langle$ ORGANIZATION $\rangle$ ). For variety, we trained three versions of Snowball for each relation, obtained by privileging precision, recall, or a combination of both via the modified $F_{1}$ measure (see Section IV), and refer to them as $S B-P, S B-R$, and $S B-C$, respectively.

Document retrieval strategies: To define the PromD and PromC retrieval strategies (Section[III), we use QXtract [1], as described in Section [II trained for each of the four extraction systems for each base relation.

5 http://www. ldc.upenn.edu 
SQOUT and baseline techniques: We compare our query processing approach, SQOUT, against 15 "baseline" techniques, each with a static choice of extraction system and document retrieval strategy. Specifically, we define four families of baseline techniques, corresponding to document retrieval strategies Scan, PromD, Const, and PromC. For each choice of retrieval strategy, we pick extraction systems (out of DIPRE, $S B-P, S B-R$, and $S B-C)$ in four different ways, namely to privilege efficiency, precision, recall, or a combination of precision and recall. We make the choice of extraction system for each relation and baseline, once and for all, using the statistics of Section V, which are computed during SQOUT's preprocessing step. For instance, for the precision baseline with PromD, we pick an extraction system for Headquarters that maximizes our precision estimate $\frac{|C(E, \operatorname{Prom} D, D)|}{|T(E, \operatorname{Prom} D, D)|}$ for that relation. The Const (PromC) baselines use the same extraction systems selected for the corresponding Scan (PromD) baselines; also, Const and PromC "degenerate" to PromD for any relation with no selection condition in a query. The prefix of a baseline name denotes whether the baseline privileges extraction system efficiency $(B: E)$, precision $(B: P)$, recall $(B: R)$, or a combination of precision and recall $(B: C)$; the suffix of the name, in turn, denotes the retrieval strategy. For example, B:P-PromD is the precision-oriented baseline that uses PromD for document retrieval. We consider all combinations except for B:E-Scan, since Scan would not be a strategy of choice for an efficiency-oriented execution.

Evaluating our optimization approach: We first run all execution strategies (i.e., SQOUT and the 15 baseline techniques) for a query and then take the union of all the tuples produced collectively, eliminating duplicates. We then verify the correctness of each tuple via the automated template-based approach of Section V-B and revisit tuples marked as correct to manually detect any suspicious tuples. (We found very few such cases.) Then, we manually check all extracted tuples that did not pass the automatic verification step. We did not perform this manual verification step for projection queries with no selection condition because their results were too large to be manageable. The final phase is to label each tuple in each query execution as correct or incorrect based on the above analysis; also, we mark as incorrect any tuple with a NULL attribute value ${ }^{7}$ such that it has a correct complete counterpart, with no NULL attribute values, that was identified by any competing execution strategies. After labeling the results for each query execution, we calculate execution time, precision, recall-computed with respect to the pool of correct tuples that all alternative executions collectively produce for the queryand goodness.

Computing environment: All our experiments were conducted on a Dell Power Edge 2650 computer server (see Table [II). We implemented our strategy in Java. We ran our experiments on an unloaded computer, restarting the Java

\footnotetext{
${ }^{6}$ If a tuple is the join of two base relation tuples, we require that each component base tuple be correct individually.

${ }^{7}$ Note that we take the outerjoin of the base relations (Section III)
}

\begin{tabular}{ll}
\hline CPU & Intel Xeon 2.4 GHz \\
RAM & 4 GB \\
Operating System & Red Hat Enterprise Linux AS release 4 (2.6 EL) \\
Runtime Environment & Java 1.5.0_12 (Sun) \\
\hline
\end{tabular}

TABLE II

COMPUTING ENVIRONMENT FOR THE EXPERIMENTS.

Virtual Machine and flushing the file buffer and CPU caches before every single execution. We used the PostgreSQL ${ }^{8}$ DBMS and, to provide a search interface to the text collections, we used Lucent? Finally, for the data cleaning step (see Section III], we used a similarity threshold of 0.9 [24].

\section{B. Experimental Results}

SQOUT vs. "Extract-then-query": As argued in the Introduction, the prevalent solution to process SQL queries over a text database is an "extract-then-query" approach, where all database documents are processed by the appropriate IE systems. To analyze the efficiency of this approach, which corresponds to the Scan retrieval strategy, we ran the $3 *_{-S \text { Scan }}$ baselines, namely $B: C$-Scan, B:P-Scan, and $B: R$-Scan, for each of our $33 \mathrm{SQL}$ queries (Section VI-A), for a total of 99 executions. Several of these executions demanded in excess of one hour to complete (single-relation executions are faster than join executions). These experiments confirm our initial observation: the extract-then-query approach is not appropriate for scenarios where efficiency is important; more generally, this approach does not adapt to user preferences on efficiency and result quality. In contrast, rather than processing all documents exhaustively, SQOUT extracts the database statistics of Table [1 in a preprocessing step, and then optimizes queries, to pick the best execution strategies for the desired efficiencyquality balance. We discuss SQOUT's preprocessing step later in this section, and now analyze the effectiveness of SQOUT's optimization approach.

SQOUT vs. Baselines: We vary the user-specified $w$ parameter (Section IV] from 0 (to privilege efficiency) to 1 (to privilege result quality). We distinguish between the queries without any selection condition, to which we refer as projection queries, and the rest, to which we refer as selection queries, because the Const and PromC retrieval strategies do not apply to projection queries.

Figure 3 shows the average goodness (Figure $3(\mathrm{a})$ ) and execution time (Figure 3(b)) of each technique over all selection queries, as a function of $w$. The values for SQOUT include the time required by the SQOUT optimization steps (Steps (1) and (2), Section IIII). SQOUT consistently has either the highest or close to the highest goodness for all values of $w$. When we privilege efficiency $(w=0)$, the SQOUT goodness is better than that for all but two baseline techniques, namely $B: C$-Const and B:P-Const. For some queries, SQOUT

$\sqrt[8]{\text { http: / / www . postgresql.org }}$

http://lucene.apache.org 


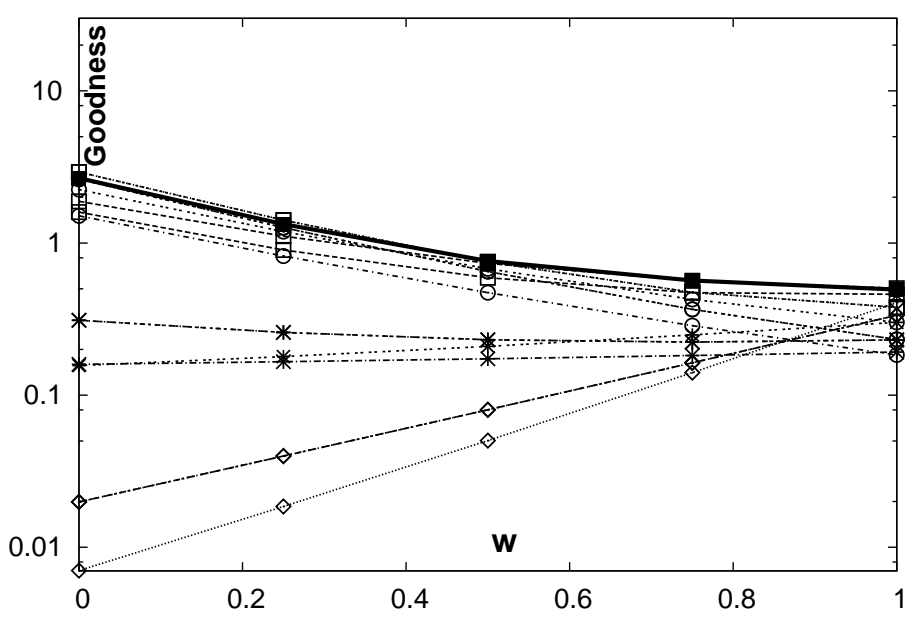

(a)

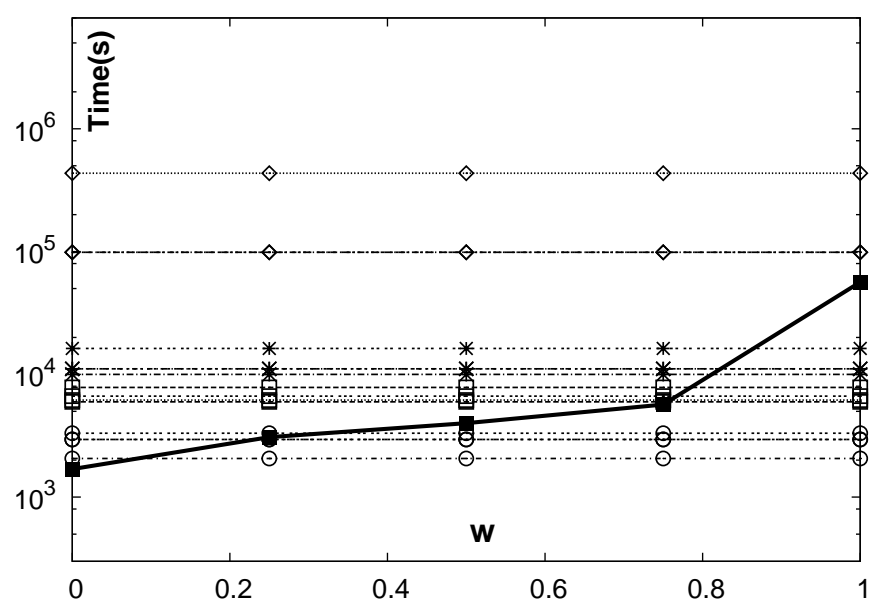

(b)

\begin{tabular}{|c|c|c|c|}
\hline 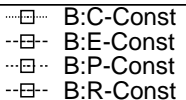 & $\begin{array}{ll}\cdots \odot \cdot & \text { B:C-PromC } \\
-\odot \cdots & \text { B:E-PromC } \\
\cdots \odot \cdots & \text { B:P-PromC } \\
\cdots \odot \cdots & \text { B:R-PromC }\end{array}$ & $\begin{array}{l}* * \cdot \text { B:C-PromD } \\
\text {-*- B:E-PromD } \\
\cdots * \cdots \text { B:P-PromD } \\
\cdots * \text { B:R-PromD }\end{array}$ & $\begin{array}{ll}\leftrightarrow & \text { B:C-Scan } \\
\cdots \diamond & \text { B:P-Scan } \\
\diamond & \text { B:R-Scan } \\
\rightarrow-\text { SQOUT }\end{array}$ \\
\hline
\end{tabular}

Fig. 3. (a) Goodness and (b) execution time over selection queries for varying $w$ (log scale).

picks execution strategies that produce no tuples. Although the SQOUT strategies are the most efficient (see Figure 3 (b)), their goodness value is zero (Definition 4.4). An example of one such query is SELECT Location FROM CompanyInfo WHERE Company $=$ 'Applied Materials': SQOUT chooses PromC as the document retrieval strategy for Headquarters, which fails to extract any answer tuples. In contrast, $B: C$-Const and $B: P$-Const use Const and manage to generate answer tuples, using a longer execution. For some other queries (e.g., SELECT Company FROM CompanyInfo WHERE CEO = 'Larry Ellison'), SQOUT chooses Const, which leads to goodness values higher than those for the $*_{-}$Prom C baselines (PromC generally retrieves fewer documents than Const, which in turn might lead to lower recall values). Therefore, the performance of SQOUT at $w=0$ lies between those of $B: C$-Const and $B: P$ -
Const and those of the *-Prom C baselines. When we privilege result quality $(w=1)$, SQOUT performs slightly worse than the best baseline, namely, B:E-Const. Finally, for projection queries SQOUT has the maximum goodness for all values of $w$. (We omit the figure because of space limitations.)

\begin{tabular}{ccccccc}
\hline & \multicolumn{3}{c}{ Extraction System } & \multicolumn{2}{c}{ Retrieval Strategy } \\
$w$ & $D I P R E$ & $S B-C$ & $S B-P$ & $S B-R$ & Const & PromC \\
\cline { 2 - 7 } 0 & 47.6 & 0 & 52.4 & 0 & 14.8 & 85.2 \\
0.5 & 35.7 & 35.7 & 0 & 28.6 & 29.6 & 70.4 \\
1.0 & 35.7 & 28.6 & 0 & 35.7 & 100 & 0 \\
\hline
\end{tabular}

TABLE III

SQOUT'S CHOICE OF EXTRACTION SYSTEM AND RETRIEVAL STRATEGY, AS A PERCENTAGE OF ALL CASES, FOR SELECTION QUERIES.

Unlike our baseline techniques, SQOUT chooses document retrieval strategies and extraction systems for each query execution. To understand these choices, Table IIII focuses on selection queries for different $w$ values. We show the fraction of times that SQOUT picks Const or PromC for base relations having an associated selection condition in the queries: for efficiency-oriented executions $(w=0)$, SQOUT mostly picks Prom $C$, whereas for quality-oriented executions $(w=1)$, SQOUT picks Const, since Const cannot miss any relevant documents for a selection query. Table IIII also shows the choice of IE systems: when efficiency is privileged, SQOUT picks DIPRE and $S B-P$, which have lower processing times; in contrast, when quality is privileged, SQOUT progresses towards selecting $S B-C$ or $S B-R$ as the choice of extraction system.

Accuracy of SQOUT's Strategy Ranking: To complete our analysis, we measured the correlation between SQOUT's ranking of strategies and the (perfect) ranking generated using the actual goodness values; a positive correlation between these rankings is a good indicator of SQOUT's ability to avoid picking the worst strategies for a query and picking the best or close to the best strategies. We obtained consistently positive values of the Spearman correlation coefficient [27], which further supports our above conclusion that SQOUT robustly picks strategies with highest or close to highest goodness.

SQOUT's Preprocessing Step: SQOUT optimizes queries based on the statistics in Table I] which are computed during a preprocessing step. With highly unoptimized code, this step required 22.6 minutes in our experiments ${ }^{10}$ This step enables SQOUT to optimize SQL queries in a user-specific manner highly effectively, as shown above. SQOUT's preprocessing overhead makes it undesirable for one family of queries, namely for queries that both (1) have no constants (i.e., where Const and PromC are not applicable) and (2) specify

${ }^{10}$ SQOUT's preprocessing step relies on the sequential sampling approach of [25. 26 (Section V. Our current implementation of this preprocessing step is not optimized for efficiency. In fact, there are many opportunities for substantially reducing the running time of this step (e.g., reusing sample documents for both the single-relation and join statistics estimation), which we will explore in our future work. 
that efficiency is not important (e.g., $w=1$ ). In such case, an extract-then-query execution is preferable: SQOUT resorts to an extract-then-query execution, but also pays the extra cost of the preprocessing overhead. However, for all other scenarios, SQOUT is indeed attractive with respect to extractthen-query. For example, for $w=0.5$ and query SELECT * FROM CompanyInfo WHERE CEO ='Eric Benhamou,' SQOUT's run time, including the preprocessing step, is $34 \%$ less than the time for the fastest extract-then-query approach, $B: P$ $S c a n$. An interesting observation is that the execution for $B: P$ Scan generated no answer tuples for this query, whereas the execution picked by SQOUT generated an answer tuple. This observation underscores the benefits of considering both the result quality as well as the execution time when selecting a query execution.

In conclusion, our estimation techniques appropriately capture the factors involved in selecting a good query execution strategy and thus produce an ordering of alternate strategies that is often close to the perfect one. Overall, we showed that our proposed optimization approach consistently picks desirable query execution strategies for the user-specified tradeoff between execution efficiency and result quality. SQOUT, as expected, privileges efficiency for low values of $w$ and result quality for high values of $w$. We have also extensively evaluated the accuracy of the efficiency and quality estimates on which SQOUT relies (Section IV). In a nutshell, the SQOUT estimates are generally close to the actual values, but the absolute errors of these estimates are often non-negligible; importantly, however, the relative rankings of the strategies according to the SQOUT efficiency and quality estimates are positively correlated-as measured using Spearman correlation tests-with the correct ranking in all cases, which explains the high goodness of the SQOUT executions. Due to space limitations, we omit further details.

\section{CONCLUSion AND Future Work}

This paper presented a principled, effective query optimization approach for simple SQL queries over text databases. Our approach relies on information extraction systems to discover the structured data that is "buried" in natural-language text, and considers both efficiency as well as query result quality when choosing an appropriate SQL query execution strategy over a text database. Many interesting research problems remain open in this area, and we plan to study them in our future work. One such problem is how to handle a richer family of SQL queries, beyond the simple selection-projectionjoin queries that we considered in this paper. Another challenging problem for future work is to explore the synergy between this paper's online extraction approach and an approach that exploits already extracted information (e.g., during earlier querying), to strike the right balance between offline computation-for persistent information needs and for the static portions of the text databases - and online extractionfor dynamic database contents and information needs.

Acknowledgments: The second author is supported by NSF CAREER grant IIS-0347903 and a Sloan fellowship. The remaining authors are supported by a generous gift from the Data Management, Exploration, and Mining Group, Microsoft Research.

\section{REFERENCES}

[1] E. Agichtein and L. Gravano, "Querying text databases for efficient information extraction," in ICDE, 2003.

[2] A. McCallum, "Information extraction: Distilling structured data from unstructured text," ACM Queue, vol. 3, no. 9, 2005.

[3] E. Agichtein and L. Gravano, "Snowball: Extracting relations from large plain-text collections," in $D L, 2000$.

[4] S. Brin, "Extracting patterns and relations from the world wide web," in $W e b D B, 1998$.

[5] M. Pasca, D. Lin, J. Bigham, A. Lifchits, and A. Jain, "Names and similarities on the web: Fact extraction in the fast lane," in $A C L, 2006$.

[6] E. Riloff, "Automatically constructing a dictionary for information extraction tasks," in IAAI, 1993.

[7] N. Kushmerick, D. Weld, and R. Doorenbos, "Wrapper induction for information extraction," in IJCAI, 1997.

[8] O. Etzioni, M. J. Cafarella, D. Downey, S. Kok, A.-M. Popescu, T. Shaked, S. Soderland, D. S. Weld, and A. Yates, "Web-scale information extraction in KnowItAll (preliminary results)," in WWW, 2004.

[9] I. Mansuri and S. Sarawagi, "A system for integrating unstructured data into relational databases," in ICDE, 2006.

[10] R. Gupta and S. Sarawagi, "Curating probabilistic databases from information extraction models," in $V L D B, 2006$.

[11] M. J. Cafarella, C. Re, D. Suciu, O. Etzioni, and M. Banko, "Structured querying of web text: A technical challenge," in CIDR, 2007.

[12] C. Kwok, O. Etzioni, and D. Weld, "Scaling question answering to the web," ACM Transactions on Information Systems, 2001.

[13] F. Chen, A. Doan, J. Yang, and R. Ramakrishnan, "Efficient information extraction over evolving text data," in ICDE, 2008.

[14] D. Ferrucci and A. Lally, "UIMA: An architectural approach to unstructured information processing in the corporate research environment," in Nat. Lang. Eng., 2004.

[15] H. Cunningham, D. Maynard, K. Bontcheva, and V. Tablan, "GATE: An architecture for development of robust HLT applications," in $A C L$, 2002.

[16] W. Shen, A. Doan, J. Naughton, and R. Ramakrishnan, "Declarative information extraction using datalog with embedded extraction predicates," in $V L D B, 2007$.

[17] E. Chu, A. Baid, T. Chen, A. Doan, and J. Naughton, "A relational approach to incrementally extracting and querying structure in unstructured data," in $V L D B, 2007$.

[18] M. Cafarella, D. Suciu, and O. Etzioni, "Navigating extracted data with schema discovery," in $W W W, 2007$.

[19] P. G. Ipeirotis, E. Agichtein, P. Jain, and L. Gravano, "To search or to crawl? Towards a query optimizer for text-centric tasks," in SIGMOD, 2006.

[20] P. G. Ipeirotis, E. Agichtein, P. Jain, and L. Gravano, "Towards a query optimizer for text-centric tasks," ACM Transactions on Database Systems, vol. 32, no. 4, Nov. 2007.

[21] A. Jain, A. Doan, and L. Gravano, "SQL queries over unstructured text databases ("poster" paper)," in ICDE, 2007.

[22] G. A. Salton and M. J. McGill, Introduction to modern information retrieval. McGraw-Hill, 1983.

[23] I. P. Fellegi and A. B. Sunter, "A theory for record linkage," Journal of the American Statistical Association, vol. 64, no. 328, Dec. 1969.

[24] L. Gravano, P. G. Ipeirotis, N. Koudas, and D. Srivastava, "Text joins in an RDBMS for web data integration," in $W W W, 2003$.

[25] P. J. Haas and A. N. Swami, "Sequential sampling procedures for query size estimation," in SIGMOD, 1992.

[26] Y. Ling and W. Sun, "An evaluation of sampling-based size estimation methods for selections in database systems," in ICDE, 1995.

[27] R. Hogg and A. Craig, Introduction to Mathematical Statistics. Macmillan, 1995.

[28] P. J. Haas, J. F. Naughton, S. Seshadri, and A. N. Swami, "Fixedprecision estimation of join selectivity," in PODS, 1993. 